\title{
Multi-wavelength observations of the GRB 080319B afterglow and the modeling constraints ${ }^{\star}$
}

\author{
S. B. Pandey ${ }^{1}$, A. J. Castro-Tirado ${ }^{2}$, M. Jelínek ${ }^{2}$, A. P. Kamble ${ }^{3}$, J. Gorosabel ${ }^{2}$, A. de Ugarte Postigo ${ }^{4}$, S. Prins ${ }^{5}$, \\ R. Oreiro ${ }^{5}$, V. Chantry ${ }^{6}$, S. Trushkin ${ }^{7}$, M. Bremer ${ }^{8}$, J. M. Winters ${ }^{8}$, A. Pozanenko ${ }^{9}$, Yu. Krugly ${ }^{14}$, I. Slyusarev ${ }^{14}$, \\ G. Kornienko ${ }^{10}$, A. Erofeeva ${ }^{10}$, K. Misra ${ }^{11}$, A. N. Ramprakash ${ }^{11}$, V. Mohan ${ }^{11}$, D. Bhattacharya ${ }^{11}$, A. Volnova ${ }^{12}$, \\ J. Plá ${ }^{13}$, M. Ibrahimov ${ }^{15}$, M. $\operatorname{Im}^{16}$, A. Volvach ${ }^{17}$, and R. A. M. J. Wijers ${ }^{3}$
}

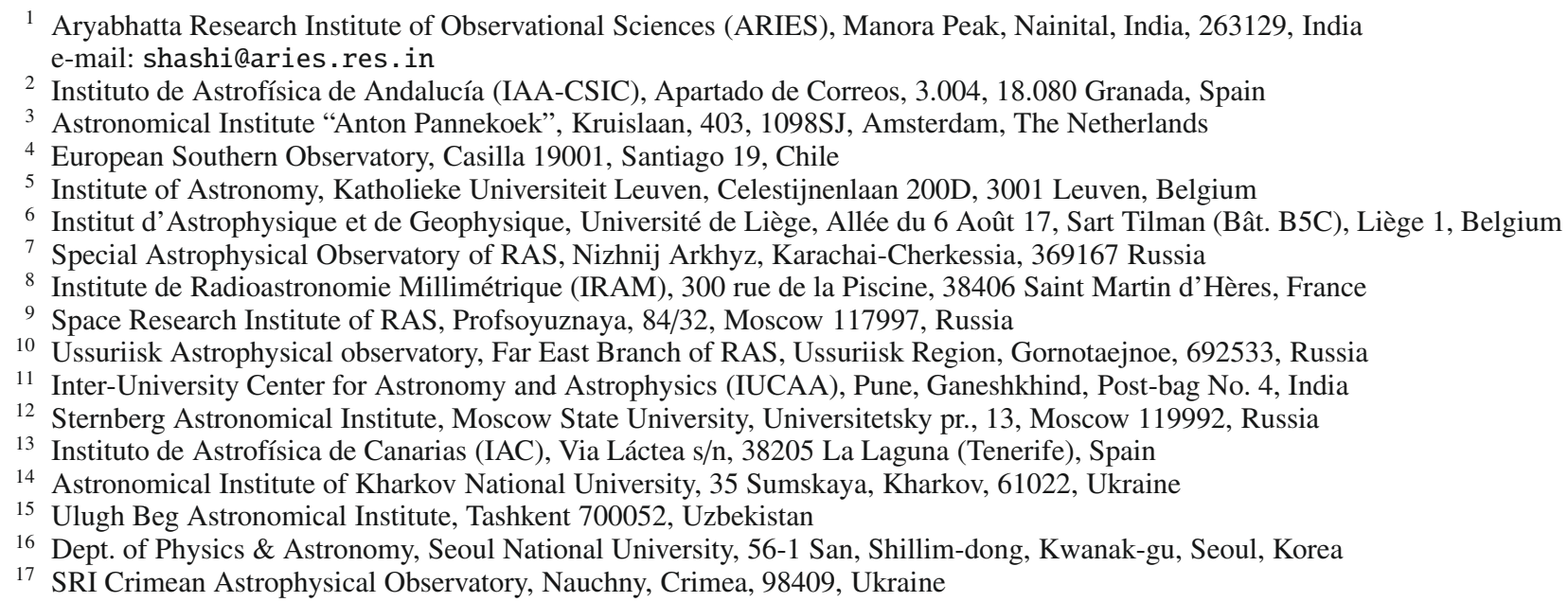

Received 11 October 2008 / Accepted 2 April 2009

\begin{abstract}
Context. We present observations of the GRB 080319B afterglow at optical, mm, and radio frequencies between a few hours and 67 days after the burst.

Aims. We attempt to understand the nature of this extraordinarily bright explosion based on the observed properties and its comparison with afterglow models.

Methods. Our observations and other published multiwavelength data were used to reconstruct the light curves and spectral energy distributions of the burst afterglow.

Results. Our results indicate that the observed features of the afterglow agrees equally well with the inter stellar matter and the stellar wind density profiles of the circumburst medium. In the case of both density profiles, the maximum synchrotron frequency $v_{\mathrm{m}}$ is below optical value and the cooling break frequency $v_{\mathrm{c}}$ is below X-rays, $\sim 10^{4} \mathrm{~s}$ after the burst. The derived value of the Lorentz factor at the time of naked-eye brightness is also $\sim 300$ for a corresponding blast-wave size of $\sim 10^{18} \mathrm{~cm}$.

Conclusions. The numerical fit to the multiwavelength afterglow data constraints the values of physical parameters and the emission mechanism of the burst.
\end{abstract}

Key words. gamma rays: bursts

\section{Introduction}

The very early-time observations of gamma-ray bursts (GRBs) during both prompt emission or early afterglow phase have been one of the major contributions during the Swift era

\footnotetext{
* Based on observations obtained with the $0.22 \mathrm{~m}$ telescope at Russia the $0.7 \mathrm{~m}$ telescope at of Kharkov University, Ukraine, the $0.8 \mathrm{~m}$ telescope at Observatorio del Teide (IAC-80), Spain the $1.2 \mathrm{~m}$ Mercator telescope at La Palma, Spain, the $1.5 \mathrm{~m}$ telescope of Maidanak observatory Uzbekistan, the $2.0 \mathrm{~m}$ IGO Telescope at IUCAA Pune, India, the $2.5 \mathrm{~m}$ NOT, the PdB millimeter interferometric array France, the RATAN-600 Radio Telescope at Russia and the RT-22 radio telescope of CrAO, Ukraine.
}

(Zhang 2007). These observations play key role in determining the physical mechanisms underlying these energetic cosmic explosions (Meśzaŕos 2006). The early-time multiwavelength observations are also very useful in constraining the afterglow models, and hence the nature of the possible progenitors and the ambient media surrounding the GRBs (Castro-Tirado et al. 1999; Piran 1999). Among the well-known examples of early observations in the pre-Swift era are GRB 990123 (Akerlof et al. 1999) and GRB 041219 (Vestrand et al. 2005) whereas GRB 050820A (Vestrand et al. 2006), GRB 060111B (Klotz et al. 2006), GRB 060210 (Stanek et al. 2007) and GRB 071010B (Wang et al. 2008) are good examples in the post-Swift era. The statistics of these examples of long-duration GRBs with very early-time 
afterglow observations have improved because of the precise onboard localization by Swift and co-ordinated observations with the ground-based robotic optical telescopes (Gehrels et al. 2004; Gomboc et al. 2006). Out of these known examples, the observed optical and $\gamma$-ray prompt emission is contemporaneous for GRB 041219 and GRB 050820A, whereas for GRB 990123 and GRB 050401, the peak of the prompt optical observations were recorded after the $\gamma$-ray emission phase.

According to the standard relativistic "fireball" model (Rees \& Meśzaŕos 1992; Meśzaŕos \& Rees 1997; Panaitescu et al. 1998), the GRB prompt emission is produced by internal shocks (Narayan et al. 1992; Rees \& Meśzaŕos 1994; Sari \& Piran 1997a,b). The observed steep decay in the early afterglow emission, which occurs between the prompt emission and the afterglow, are also explained in terms of "high-latitude emission" (Nousek et al. 2006) in the case of Swift GRBs, irrespective of the type of shock or the radiative process involved. The very early-time optical observations of several GRB afterglows are explained in terms of having a reverse shock (Kobayashi 2000) or/and forward shock (Rees \& Meśzaŕos 1992; Katz 1994) origin. The overall afterglow behavior of long-duration GRBs, including the aforementioned examples, are explained in terms of the "collapse of very massive stars"i, i.e., so-called "collapsars" as the most favored progenitor (MacFadyen \& Woosley 1999). By now, the majority of long-duration GRB afterglows have been explained in terms of constant ambient density i.e., inter stellar medium (ISM, $\rho \propto r^{0}$ ) models (Sari, et al. 1998; Wijers \& Galama 1999; Panaitescu \& Kumar 2002), although a Stellar Wind Medium $\left(W M, \rho \propto r^{-2}\right.$ ) profile (Chevalier \& Lee 2000a,b; Li \& Chevalier 2001) is the natural result of massivestar environments (Zhang 2007), where $\rho$ and $r$ are the ambient density and the distance from the center of the progenitor star, respectively. The value of ambient density is constrained by the parameters "number density" $n$ and the "wind parameter" $A_{*}$, respectively for the $I S M$ and $W M$ models. There are also certain cases of long-duration GRB afterglows that have been explained in terms of both density profiles in the form of the transition from $W M$ density profile at early times to ISM density profile at later epochs of observations, e.g., GRB 050904 (Gendre et al. 2007) and GRB 050319 (Kamble et al. 2007).

GRB 080319B was triggered (trigger $=306757$ ) by SwiftBAT (15-350 keV) at $T_{0}=06: 12: 49$ UT on March 19, 2008 (Racusin et al. 2008a) and was simultaneously detected by the Konus-Wind (20 keV-15 MeV) satellite (Golenetskii et al. 2008). The optical emission started $2.75 \pm 5 \mathrm{~s}$ after the BAT trigger, and was captured by the wide-field robotic telescope "Pi of the Sky" (Cwiok et al. 2007, 2008) and also TORTORA (Molinari et al. 2006; Racusin 2008b) and Raptor-Q (Wozniak et al. 2009) at later epochs. For a few seconds after the burst, the observed prompt optical flash of GRB 080319B was visible even to the unaided eye in dark skies (peaking at a visual magnitude of 5.3 around $18 \mathrm{~s}$ after the onset of the burst, observed by TORTORA), breaking the record of a handful of known cases in which bright optical-NIR prompt emissions have been observed (Jelinek et al. 2007). Swift-UVOT slewed towards the burst $51 \mathrm{~s}$ after the trigger (Holland \& Racusin 2008) and later many other ground and space-based multiwavelength facilities joined in, as summarized in detail by Bloom et al. (2009), Racusin et al. (2008b), and references therein.

The measured redshift value of the burst $(z=0.937$, Vreeswijk et al. 2008) corresponds to a luminosity distance of $d_{\mathrm{L}}=6.01 \times 10^{3} \mathrm{Mpc}$ (for the cosmological parameters $\left.H_{0}=71 \mathrm{~km} \mathrm{~s}^{-1} \mathrm{Mpc}^{-1}, \Omega_{M}=0.27, \Omega_{\Lambda}=0.73\right)$ and an equivalent isotropic energy in $\gamma$-rays of $E_{\gamma \text {,iso }}=1.4 \times 10^{54} \mathrm{erg}$
Table 1. Log of milli meter-wave and radio observations of the GRB 080319B afterglow.

\begin{tabular}{ccccc}
\hline \hline $\begin{array}{c}\text { Start } \\
\text { time (UT) }\end{array}$ & $\begin{array}{c}\text { End } \\
\text { time }(\mathrm{UT})\end{array}$ & $\begin{array}{c}\text { Frequency } \\
(\mathrm{GHz})\end{array}$ & $\begin{array}{c}\text { Flux } \\
\text { center }(\mathrm{mJy})\end{array}$ & Telescope \\
\hline 2008 Mar. 20.0 & Mar. 20.5 & 97.0 & $+0.41 \pm 0.12$ & IRAM \\
2008 Mar. 23.0 & Mar. 23.5 & 97.0 & $+0.35 \pm 0.19$ & IRAM \\
2008 Mar. 27.0 & Mar. 27.5 & 97.0 & $+0.20 \pm 0.09$ & IRAM \\
& & & & \\
2008 Mar. 28.0 & Mar. 28.5 & 4.8 & $<4.0$ & RATAN \\
2008 Mar. 30.0 & Mar. 30.5 & 4.8 & $<4.0$ & RATAN \\
2008 Apr. 08.0 & Apr. 08.5 & 4.8 & $<3.0$ & RATAN \\
& & & & \\
2008 May 25.0 & May 25.5 & 2.0 & $<3.0$ & RT-22 \\
2008 May 25.0 May 25.5 & 8.0 & $<3.0$ & RT-22 \\
\hline
\end{tabular}

(20 keV-7 MeV), which is among the highest energy ever measured for GRBs. The inferred high luminosity of the burst after correcting to the Galactic and host galaxy extinction in the burst direction (Wozniak et al. 2009) implies that such an event could easily have been detected at redshift $z=17$ with meter-class telescopes in the $K$-band (Bloom et al. 2009; Sagar 2005). The observed value of the optical flash flux density of GRB 080319B ( $20 \mathrm{Jy})$ is also about 4 orders of magnitude higher than the peak flux density in $\gamma$-rays $(\sim 14 \mathrm{mJy})$, the highest ever observed for GRBs (Yu et al. 2008). The significant excess of prompt optical flux compared to the extrapolated $\gamma$-ray spectrum implies that different emission components related to the two observed frequencies (Racusin et al. 2008b).

Thus, it is clear that multiwavelength afterglow observations of GRB 080319B provide unique opportunity to study the nature of this energetic cosmic explosion in detail. The observed spectral and temporal coverage of the afterglow imply that the burst is one of the most well-known examples for testing theoretical afterglow models (Pandey et al. 2003; Resmi et al. 2005). In this paper, we summarize the radio, milli meter-wave $(\mathrm{mm})$, and optical observations of the afterglow in Sect. 2. The afterglow properties are discussed in Sect. 3. The modeling of the multiwavelength afterglow data and the derived parameters are described in Sect. 4. In the last section, we summarize our results in the context of observed and modeled parameters of the burst.

\section{Observations}

\subsection{Milli meter-wave and radio observations}

Observations of GRB 080319B afterglow at millimeter frequencies were performed with the Plateau de Bure (PdB) Interferometer (Guilloteau et al. 1992) in a six-antenna extended configuration on the dates listed in Table 1. The afterglow was detected in the first round of observations starting 0.5 days after the burst and upper limits were established at two later epochs. Radio observations were also performed using RATAN-600 at $4.8 \mathrm{GHz}$ between 9 and 19 days after the burst and the afterglow was not detected. The afterglow was also monitored using the radio telescope $\mathrm{RT}-22 / \mathrm{CrAO}$ at 2.0 and $8.0 \mathrm{GHz}$ on 25th May 2008. The upper limits $(3 \sigma)$ inferred from RATAN-600 and RT22/CrAO observations are also listed in Table 1. 


\subsection{Optical observations}

Our optical observations were performed using several telescopes (0.22 m SR-22; 0.7 m AZT-8; 0.8 m IAC; $1.2 \mathrm{~m}$ Mercator, 1.5 m Maidanak (AZT-22), 2.0 m IGO, 2.5 m NOT(+ALFOSC)) in the far east of Russia, Europe, Middle Asia and India starting from 0.5 day to 19 days after the burst. IRAF and DAOPHOT softwares were used to perform data reduction using standard techniques. The $B, V, R$, and $I$ data were calibrated using the nearby secondary standards as given in Henden (2008). The Gunn $r$ data were calibrated using nearby calibrators. The log of our optical observations along with the details are given in Table 2.

\section{Properties of the burst}

In this section, we discuss the observed temporal properties of the burst during the prompt emission and during the afterglow phase.

\subsection{Prompt emission phase}

The prompt optical light curve appears to be correlated with the $\gamma$-ray light curve, because of the high time-resolution (Racusin et al. 2008b). This correlation suggests that radiation at both frequencies originate in the same physical source based on the assumptions of internal shock model (Meśzaŕos \& Rees 1999; Kumar \& Panaitescu 2008). There is no considerable evolution in the X-ray hardness ratio from very early to late times of the observations with an average photon index $\Gamma=-1.81 \pm 0.04$ (Bloom et al. 2009). There are no multi-band optical observations during the prompt emission phase of the burst. The observed spectral indices around $430 \mathrm{~s}$ and $875 \mathrm{~s}$ after the burst also show negligible evolution at optical frequencies (Bloom et al. 2009). The reverse shock or forward shock origin of the observed prompt optical emission is excluded based on the observed values of temporal decay indices from being too steep than expected (Sari, et al. 1998; Kobayashi 2000). The observed constancy of the optical pulse width with time during the prompt emission phase (Ramirez-Ruiz \& Fenimore 2000) also does not support the reverse shock origin. The temporal coincidence between the prompt optical and $\gamma$-rays of the burst also indicate that they might have originated in the same emitting region or two regions sharing the same dynamical behavior (Yu et al. 2008). The correlation of spectral lag evolution with the observed prompt optical emission of GRB 080319B also shows that they originate from the same astrophysical origin and that the respective radiation mechanisms were dynamically coupled (Stamatikos et al. 2009). Furthermore, the significant excess of the prompt optical flux in comparison to the $\gamma$-ray spectrum extrapolated to optical frequencies, is possibly indicative of two different emission components for the two observed frequencies (but see Kumar \& Panaitescu 2008).

\subsection{Multi-frequency evolution of the afterglow}

According to the standard fireball model, the GRB afterglow is expected to be synchrotron radiation with the observed flux $f \propto v^{\beta} t^{\alpha}$, for the regions without spectral breaks, where the values of power-law temporal decay index $(\alpha)$ and spectral index $(\beta)$ are related to each other and can be used to understand the afterglow evolution (Sari, et al. 1998; Sari, et al. 1999). The rich multi-frequency data of the afterglow of GRB 080319B available in the literature and the data from the present work are used to measure the indices $\alpha$ and $\beta$ of the afterglow. The values of the temporal decay indices have been derived using empirical broken power-law relations by minimization of $\chi^{2}$ as described in Granot \& Sari (2002) (their Eqs. (1) and (4)). The bright optical flash $(10<t<100 \mathrm{~s})$ rises to its peak brightness $(V \sim 5.32 \pm 0.04)$ with $\alpha_{V 1}=4.64 \pm 0.67$, and after about $50 \mathrm{~s}$ decays with $\alpha_{V 2}=-4.41 \pm 0.10$. After $\sim 100 \mathrm{~s}$, the decay of the optical afterglow could be described as a broken power-law with $\alpha_{V 3}=-2.33 \pm 0.23, \alpha_{V 4}=-1.31 \pm 0.02$ with the break occurring at $\sim 784.9 \pm 304.4 \mathrm{~s}$ after the burst. The X-ray afterglow light curve is described by a triple broken power-law with $\alpha_{\mathrm{X} 1}=-1.40 \pm 0.01, \alpha_{\mathrm{X} 2}=-1.94 \pm 0.12$, $\alpha_{\mathrm{X} 3}=-1.14 \pm 0.09$, and $\alpha_{\mathrm{X} 4}=-2.67 \pm 0.74$ and the breaks happening at $t_{\mathrm{b}, \mathrm{X} 1}=2583.39 \pm 871 \mathrm{~s}, t_{\mathrm{b}, \mathrm{X} 2}=(3.99 \pm 0.89) \times 10^{4} \mathrm{~s}$, and $t_{\mathrm{b}, \mathrm{X} 3}=(1.0 \pm 0.29) \times 10^{6} \mathrm{~s}$ with $\chi^{2} / d o f=975 / 710=1.37$. The derived value of the X-ray temporal index $\alpha_{\mathrm{X} 4}$ and the corresponding break time are in agreement with those derived by Tanvir et al. (2009) using late-time X-ray and optical data, which provide evidence in favor of jet-break around $\sim 10^{6} \mathrm{~s}$ after the burst.

\section{Discussion}

In this section, we discuss about the burst ambient media and the numerical fit to the afterglow data.

\subsection{Surrounding medium}

The closure relations between $\alpha$ and $\beta$ can also be used to infer the density profile of the circumburst medium or to distinguish between theoretical afterglow models such as ISM and WM (Price et al. 2002; Starling et al. 2008). For radiation produced by a shock interacting with the ISM circumburst medium, the expected closure relation is $\alpha-1.5 \beta=0.0$ in a spectral regime $v_{\mathrm{m}}<v<v_{\mathrm{c}}$, where $v_{\mathrm{m}}$ and $v_{\mathrm{c}}$ are the maximum synchrotron and cooling frequencies, respectively. In the case of the WM circumburst medium and a spectral regime $v_{\mathrm{m}}<v<v_{\mathrm{c}}$, the closure relation is $\alpha-1.5 \beta=0.5$. If $\nu_{\mathrm{m}}<v_{\mathrm{c}}<v$, then $\alpha-1.5 \beta=-0.5$ is expected irrespective of whether the density profile is ISM or $W M$. At early times $(t \leq 2000 \mathrm{~s})$, the optical afterglow is dominated by emission caused by the reverse shock, and at late times $\left(t \geq 10^{5} \mathrm{~s}\right)$ it is clearly dominated by that due to the forward shock. The values of $\beta$ estimated by Bloom et al. (2009) and the values of $\alpha$ inferred from optical light curves at $t>10^{5} \mathrm{~s}$ are consistent with the closure relations for both the density profiles, $I S M$ and $W M$ in the spectral regime $v_{\mathrm{m}}<v_{\text {optical }}<v_{\mathrm{c}}$.

It is also clear that the temporal decay index of the optical afterglow between $100 \mathrm{~s}$ and $1000 \mathrm{~s}(\alpha \sim-2.3)$ is higher than expected for a forward shock scenario $(\alpha \sim-0.8)$ interacting with the circumburst medium (Sari, et al. 1998). However, this steep decay would be expected in the case of a reverse shock interacting with the ejected shell. The radiation produced by the interaction of the forward shock with the circumburst medium would then dominate the afterglow after the radiation from reverse shock has diminished considerably, which in this case could happen after $10^{5} \mathrm{~s}$. Thus, this optical afterglow could be explained using the reverse-shock and forward-shock interactions with the circumburst medium.

\subsection{Numerical model fits to the data}

We fitted the afterglow using both a reverse shock (RS) model and a forward shock (FS) model, and assumed that the 
Table 2. Log of optical-NIR observations of the afterglow of GRB 080319B.

\begin{tabular}{|c|c|c|c|c|c|c|}
\hline$U T$ start [d] & $T-T_{0}[\mathrm{~d}]$ & $T_{\exp }$ & Filter & mag & err ${ }_{\text {mag }}$ & Telescope \\
\hline 2008 Mar. 19.4616 & 0.2027 & $1200 \mathrm{~s}$ & none & $>15.5$ & & $0.22 \mathrm{~m} \mathrm{SR}-22$ \\
\hline 2008 Mar. 19.7642 & 0.5052 & $600 \mathrm{~s}$ & $R$ & 19.07 & 0.19 & $0.7 \mathrm{~m}$ AZT-8 \\
\hline 2008 Mar. 19.7721 & 0.5132 & $600 \mathrm{~s}$ & $R$ & 19.26 & 0.27 & 0.7 m AZT-8 \\
\hline 2008 Mar. 19.7800 & 0.5211 & $600 \mathrm{~s}$ & $R$ & 19.74 & 0.50 & 0.7 m AZT-8 \\
\hline 2008 Mar. 19.7911 & 0.5322 & $600 \mathrm{~s}$ & $R$ & 19.96 & 0.39 & 0.7 m AZT-8 \\
\hline 2008 Mar. 19.7974 & 0.5385 & $600 \mathrm{~s}$ & $R$ & 19.60 & 0.34 & 0.7 m AZT-8 \\
\hline 2008 Mar. 19.8055 & 0.5466 & $900 \mathrm{~s}$ & $R$ & 19.60 & 0.20 & 0.7 m AZT-8 \\
\hline 2008 Mar. 19.8174 & 0.5585 & $900 \mathrm{~s}$ & $R$ & 19.40 & 0.15 & $0.7 \mathrm{~m}$ AZT-8 \\
\hline 2008 Mar. 19.8285 & 0.5696 & $900 \mathrm{~s}$ & $R$ & 19.89 & 0.28 & 0.7 m AZT-8 \\
\hline 2008 Mar. 19.8470 & 0.5881 & $900 \mathrm{~s}$ & $R$ & 19.82 & 0.23 & 0.7 m AZT-8 \\
\hline 2008 Mar. 19.8557 & 0.5968 & $900 \mathrm{~s}$ & $R$ & 20.06 & 0.19 & 0.7 m AZT-8 \\
\hline 2008 Mar. 19.8630 & 0.6041 & $900 \mathrm{~s}$ & $R$ & 19.88 & 0.23 & 0.7 m AZT-8 \\
\hline 2008 Mar. 19.8888 & 0.6299 & $720 \mathrm{~s}$ & $R$ & 20.00 & 0.27 & 0.7 m AZT-8 \\
\hline 2008 Mar. 19.8980 & 0.6391 & $720 \mathrm{~s}$ & $R$ & 19.52 & 0.16 & 0.7 m AZT-8 \\
\hline 2008 Mar. 19.9071 & 0.6482 & $720 \mathrm{~s}$ & $R$ & 20.09 & 0.28 & 0.7 m AZT-8 \\
\hline 2008 Mar. 19.9139 & 0.6550 & $720 s$ & $R$ & 19.80 & 0.24 & $0.7 \mathrm{~m}$ AZT-8 \\
\hline 2008 Mar. 19.9234 & 0.6645 & $900 \mathrm{~s}$ & $R$ & 19.88 & 0.27 & $0.7 \mathrm{~m}$ AZT-8 \\
\hline 2008 Mar. 19.9354 & 0.6765 & $900 \mathrm{~s}$ & $R$ & 20.19 & 0.40 & $0.7 \mathrm{~m}$ AZT-8 \\
\hline 2008 Mar. 19.9497 & 0.6908 & $900 \mathrm{~s}$ & $R$ & 19.54 & 0.15 & $0.7 \mathrm{~m} \mathrm{AZT}-8$ \\
\hline 2008 Mar. 19.9625 & 0.7036 & $900 \mathrm{~s}$ & $R$ & 20.16 & 0.44 & $0.7 \mathrm{~m} \mathrm{AZT-8}$ \\
\hline 2008 Mar. 19.9752 & 0.7163 & $720 \mathrm{~s}$ & $R$ & 20.47 & 0.25 & $0.7 \mathrm{~m}$ AZT-8 \\
\hline 2008 Mar. 19.9843 & 0.7254 & $960 \mathrm{~s}$ & $R$ & 20.00 & 0.37 & $0.7 \mathrm{~m} \mathrm{AZT-8}$ \\
\hline 2008 Mar. 19.9959 & 0.7370 & $900 \mathrm{~s}$ & $R$ & 20.03 & 0.37 & $0.7 \mathrm{~m} \mathrm{AZT-8}$ \\
\hline 2008 Mar. 20.0127 & 0.7538 & $720 \mathrm{~s}$ & $R$ & 20.01 & 0.22 & $0.7 \mathrm{~m} \mathrm{AZT-8}$ \\
\hline 2008 Mar. 20.0196 & 0.7607 & $720 \mathrm{~s}$ & $R$ & 19.83 & 0.21 & $0.7 \mathrm{~m}$ AZT-8 \\
\hline 2008 Mar. 20.0664 & 0.8075 & $720 \mathrm{~s}$ & $R$ & 19.94 & 0.23 & $0.7 \mathrm{~m}$ AZT- 8 \\
\hline 2008 Mar. 20.0755 & 0.8166 & $720 \mathrm{~s}$ & $R$ & 20.22 & 0.26 & $0.7 \mathrm{~m} \mathrm{AZT-8}$ \\
\hline 2008 Mar. 20.0848 & 0.8259 & $900 \mathrm{~s}$ & $R$ & 20.28 & 0.24 & $0.7 \mathrm{~m} \mathrm{AZT-8}$ \\
\hline 2008 Mar. 20.0959 & 0.8370 & $900 \mathrm{~s}$ & $R$ & 19.74 & 0.16 & $0.7 \mathrm{~m} \mathrm{AZT-8}$ \\
\hline 2008 Mar. 20.1069 & 0.8480 & $600 \mathrm{~s}$ & $R$ & 19.56 & 0.20 & $0.7 \mathrm{~m} \mathrm{AZT-8}$ \\
\hline 2008 Mar. 19.8319 & 0.5730 & $600 \mathrm{~s}$ & $V$ & 19.60 & 0.70 & $0.7 \mathrm{~m}$ AZT-8 \\
\hline 2008 Mar. 20.0870 & 0.8351 & $1200 \mathrm{~s}$ & $I$ & 19.57 & 0.08 & $0.8 \mathrm{~m}$ IAC 80 \\
\hline 2008 Mar. 19.9924 & 0.7457 & $4 \times 300 \mathrm{~s}$ & $R$ & 19.82 & 0.07 & $0.8 \mathrm{~m}$ IAC 80 \\
\hline 2008 Mar. 20.0557 & 0.8003 & $600 \mathrm{~s}$ & $R$ & 19.94 & 0.11 & $0.8 \mathrm{~m}$ IAC 80 \\
\hline 2008 Mar. 20.1067 & 0.8548 & $2 \times 600 \mathrm{~s}$ & $R$ & 20.15 & 0.08 & $0.8 \mathrm{~m}$ IAC 80 \\
\hline 2008 Mar. 20.1294 & 0.8778 & $2 \times 600 \mathrm{~s}$ & $R$ & 20.14 & 0.09 & $0.8 \mathrm{~m}$ IAC 80 \\
\hline 2008 Mar. 20.2454 & 0.9972 & $3 \times 600 \mathrm{~s}$ & $R$ & 20.50 & 0.08 & $0.8 \mathrm{~m}$ IAC 80 \\
\hline 2008 Mar. 22.0730 & 2.8355 & $6 \times 600 s$ & $R$ & 22.03 & 0.27 & $0.8 \mathrm{~m}$ IAC 80 \\
\hline 2008 Mar. 20.0252 & 0.7814 & $(1200+600) \mathrm{s}$ & V & 20.39 & 0.10 & $0.8 \mathrm{~m}$ IAC 80 \\
\hline 2008 Mar. 20.0648 & 0.8165 & $3 \times 600 \mathrm{~s}$ & $B$ & 20.89 & 0.12 & $0.8 \mathrm{~m}$ IAC 80 \\
\hline 2008 Mar. 20.1703 & 0.9149 & $600 \mathrm{~s}$ & $B$ & 20.75 & 0.20 & $0.8 \mathrm{~m}$ IAC 80 \\
\hline 2008 Mar. 19.9361 & 0.6985 & $120 \mathrm{~s}$ & $r$ & 19.56 & 0.17 & $1.2 \mathrm{~m}$ Mercator \\
\hline 2008 Mar. 19.9613 & 0.7031 & $120 \mathrm{~s}$ & $r$ & 19.28 & 0.19 & $1.2 \mathrm{~m}$ Mercator \\
\hline 2008 Mar. 19.9631 & 0.7049 & $120 \mathrm{~s}$ & $r$ & 19.74 & 0.15 & $1.2 \mathrm{~m}$ Mercator \\
\hline 2008 Mar. 19.9649 & 0.7067 & $120 \mathrm{~s}$ & $r$ & 19.37 & 0.11 & $1.2 \mathrm{~m} \mathrm{Mercator}$ \\
\hline 2008 Mar. 19.9667 & 0.7085 & $120 \mathrm{~s}$ & $r$ & 19.93 & 0.15 & $1.2 \mathrm{~m} \mathrm{Mercator}$ \\
\hline 2008 Mar. 19.9681 & 0.7095 & $60 \mathrm{~s}$ & $r$ & 19.53 & 0.11 & $1.2 \mathrm{~m}$ Mercator \\
\hline 2008 Mar. 19.9691 & 0.7106 & $60 \mathrm{~s}$ & $r$ & 20.07 & 0.21 & $1.2 \mathrm{~m}$ Mercator \\
\hline 2008 Mar. 19.9702 & 0.7117 & $60 \mathrm{~s}$ & $r$ & 19.41 & 0.10 & $1.2 \mathrm{~m}$ Mercator \\
\hline 2008 Mar. 19.9713 & 0.7127 & $60 \mathrm{~s}$ & $r$ & 19.66 & 0.20 & $1.2 \mathrm{~m} \mathrm{Mercator}$ \\
\hline 2008 Mar. 19.9723 & 0.7138 & $60 \mathrm{~s}$ & $r$ & 19.82 & 0.15 & $1.2 \mathrm{~m}$ Mercator \\
\hline 2008 Mar. 19.9734 & 0.7148 & $60 \mathrm{~s}$ & $r$ & 19.68 & 0.09 & $1.2 \mathrm{~m} \mathrm{Mercator}$ \\
\hline 2008 Mar. 19.9745 & 0.7159 & $60 \mathrm{~s}$ & $r$ & 19.61 & 0.09 & $1.2 \mathrm{~m}$ Mercator \\
\hline 2008 Mar. 19.9759 & 0.7177 & $120 \mathrm{~s}$ & $r$ & 19.57 & 0.12 & $1.2 \mathrm{~m}$ Mercator \\
\hline 2008 Mar. 19.9777 & 0.7195 & $120 \mathrm{~s}$ & $r$ & 19.54 & 0.09 & $1.2 \mathrm{~m}$ Mercator \\
\hline 2008 Mar. 19.9794 & 0.7212 & $120 \mathrm{~s}$ & $r$ & 19.62 & 0.07 & $1.2 \mathrm{~m}$ Mercator \\
\hline 2008 Mar. 19.9812 & 0.7230 & $120 \mathrm{~s}$ & $r$ & 19.83 & 0.09 & $1.2 \mathrm{~m}$ Mercator \\
\hline 2008 Mar. 19.9830 & 0.7248 & $120 \mathrm{~s}$ & $r$ & 19.58 & 0.06 & $1.2 \mathrm{~m}$ Mercator \\
\hline 2008 Mar. 19.9848 & 0.7266 & $120 \mathrm{~s}$ & $r$ & 19.65 & 0.07 & $1.2 \mathrm{~m} \mathrm{Mercator}$ \\
\hline 2008 Mar. 19.9865 & 0.7283 & $120 \mathrm{~s}$ & $r$ & 19.67 & 0.07 & $1.2 \mathrm{~m}$ Mercator \\
\hline
\end{tabular}


Table 2. continued.

\begin{tabular}{|c|c|c|c|c|c|c|}
\hline$\overline{U U T \text { start }[\mathrm{d}]}$ & $T-T_{0}[\mathrm{~d}]$ & $\overline{\bar{T}} \overline{\text { exp }}$ & Filter & mag & err $_{\text {mag }}$ & Telescope \\
\hline 2008 Mar. 19.9883 & 0.7301 & $120 \mathrm{~s}$ & $\bar{r}$ & 19.78 & 0.08 & 1.2 m Mercator \\
\hline 2008 Mar. 19.9900 & 0.7318 & $120 \mathrm{~s}$ & $r$ & 19.51 & 0.07 & $1.2 \mathrm{~m}$ Mercator \\
\hline 2008 Mar. 19.9918 & 0.7336 & $120 \mathrm{~s}$ & $r$ & 19.66 & 0.08 & $1.2 \mathrm{~m}$ Mercator \\
\hline 2008 Mar. 19.9936 & 0.7354 & $120 \mathrm{~s}$ & $r$ & 19.47 & 0.05 & $1.2 \mathrm{~m}$ Mercator \\
\hline 2008 Mar. 19.9953 & 0.7371 & $120 \mathrm{~s}$ & $r$ & 19.62 & 0.06 & $1.2 \mathrm{~m}$ Mercator \\
\hline 2008 Mar. 19.9971 & 0.7389 & $120 \mathrm{~s}$ & $r$ & 19.46 & 0.05 & $1.2 \mathrm{~m}$ Mercator \\
\hline 2008 Mar. 19.9989 & 0.7407 & $120 \mathrm{~s}$ & $r$ & 19.62 & 0.06 & $1.2 \mathrm{~m}$ Mercator \\
\hline 2008 Mar. 20.1446 & 0.8871 & $120 \mathrm{~s}$ & $r$ & 19.76 & 0.07 & $1.2 \mathrm{~m}$ Mercator \\
\hline 2008 Mar. 20.1464 & 0.8888 & $120 \mathrm{~s}$ & $r$ & 19.95 & 0.08 & $1.2 \mathrm{~m}$ Mercator \\
\hline 2008 Mar. 20.1481 & 0.8906 & $120 \mathrm{~s}$ & $r$ & 20.14 & 0.10 & $1.2 \mathrm{~m}$ Mercator \\
\hline 2008 Mar. 20.1500 & 0.8925 & $120 \mathrm{~s}$ & $r$ & 19.78 & 0.07 & $1.2 \mathrm{~m}$ Mercator \\
\hline 2008 Mar. 20.1517 & 0.8942 & $120 \mathrm{~s}$ & $r$ & 20.04 & 0.11 & $1.2 \mathrm{~m}$ Mercator \\
\hline 2008 Mar. 20.1535 & 0.8960 & $120 \mathrm{~s}$ & $r$ & 19.98 & 0.10 & $1.2 \mathrm{~m}$ Mercator \\
\hline 2008 Mar. 20.1554 & 0.8979 & $120 \mathrm{~s}$ & $r$ & 19.85 & 0.08 & $1.2 \mathrm{~m}$ Mercator \\
\hline 2008 Mar. 20.1572 & 0.8997 & $120 \mathrm{~s}$ & $r$ & 19.85 & 0.12 & $1.2 \mathrm{~m}$ Mercator \\
\hline 2008 Mar. 20.1589 & 0.9014 & $120 \mathrm{~s}$ & $r$ & 19.87 & 0.13 & $1.2 \mathrm{~m}$ Mercator \\
\hline 2008 Mar. 20.1607 & 0.9032 & $120 \mathrm{~s}$ & $r$ & 19.87 & 0.11 & $1.2 \mathrm{~m} \mathrm{Mercator}$ \\
\hline 2008 Mar. 20.1625 & 0.9049 & $120 \mathrm{~s}$ & $r$ & 19.92 & 0.09 & $1.2 \mathrm{~m}$ Mercator \\
\hline 2008 Mar. 20.1642 & 0.9067 & $120 \mathrm{~s}$ & $r$ & 19.96 & 0.11 & $1.2 \mathrm{~m}$ Mercator \\
\hline 2008 Mar. 20.1660 & 0.9085 & $120 \mathrm{~s}$ & $r$ & 20.04 & 0.10 & $1.2 \mathrm{~m}$ Mercator \\
\hline 2008 Mar. 20.1678 & 0.9103 & $120 \mathrm{~s}$ & $r$ & 19.84 & 0.08 & $1.2 \mathrm{~m} \mathrm{Mercator}$ \\
\hline 2008 Mar. 20.1695 & 0.9120 & $120 \mathrm{~s}$ & $r$ & 19.73 & 0.09 & $1.2 \mathrm{~m}$ Mercator \\
\hline 2008 Mar. 20.1713 & 0.9138 & $120 \mathrm{~s}$ & $r$ & 19.84 & 0.11 & $1.2 \mathrm{~m} \mathrm{Mercator}$ \\
\hline 2008 Mar. 20.1731 & 0.9156 & $120 \mathrm{~s}$ & $r$ & 19.92 & 0.10 & $1.2 \mathrm{~m}$ Mercator \\
\hline 2008 Mar. 20.1748 & 0.9173 & $120 \mathrm{~s}$ & $r$ & 20.01 & 0.12 & $1.2 \mathrm{~m}$ Mercator \\
\hline 2008 Mar. 20.1766 & 0.9191 & $120 \mathrm{~s}$ & $r$ & 19.91 & 0.10 & $1.2 \mathrm{~m}$ Mercator \\
\hline 2008 Mar. 20.1784 & 0.9208 & $120 \mathrm{~s}$ & $r$ & 20.08 & 0.09 & $1.2 \mathrm{~m}$ Mercator \\
\hline 2008 Mar. 20.1801 & 0.9226 & $120 \mathrm{~s}$ & $r$ & 19.71 & 0.07 & $1.2 \mathrm{~m}$ Mercator \\
\hline 2008 Mar. 20.1819 & 0.9244 & $120 \mathrm{~s}$ & $r$ & 19.87 & 0.09 & $1.2 \mathrm{~m}$ Mercator \\
\hline 2008 Mar. 20.1837 & 0.9261 & $120 \mathrm{~s}$ & $r$ & 19.83 & 0.07 & $1.2 \mathrm{~m}$ Mercator \\
\hline 2008 Mar. 20.1854 & 0.9279 & $120 \mathrm{~s}$ & $r$ & 19.84 & 0.09 & $1.2 \mathrm{~m}$ Mercator \\
\hline 2008 Mar. 21.8618 & 2.6029 & $5760 \mathrm{~s}$ & $I$ & 21.22 & 0.15 & $1.5 \mathrm{~m}$ AZT-22 \\
\hline 2008 Mar. 21.8781 & 2.6192 & $5610 \mathrm{~s}$ & $R$ & 21.81 & 0.26 & $1.5 \mathrm{~m}$ AZT-22 \\
\hline 2008 Apr. 07.0086 & 18.7479 & $7200 \mathrm{~s}$ & $R$ & $>23.6$ & & $1.5 \mathrm{~m}$ AZT-22 \\
\hline 2008 Mar. 19.9179 & 0.6590 & $3 \times 600 \mathrm{~s}$ & $R$ & 19.66 & 0.09 & $2.0 \mathrm{~m} \mathrm{IGO}$ \\
\hline 2008 Mar. 27.9511 & 8.6524 & $28 \times 120 \mathrm{~s}$ & $R$ & $>23.0$ & & $2.5 \mathrm{~m} \mathrm{NOT}$ \\
\hline
\end{tabular}

radiation mechanism is synchrotron following the standard Fireball scenario (Rees \& Meśzaŕos 1992; Meśzaŕos \& Rees 1994; Kobayashi 2000). As shown in Fig. 1, the multi-band optical afterglow is reproduced well by this model, whereas X-ray observations cannot be reproduced by the model. Our best-fit model is consistent with no jet break in the optical afterglow, and introducing a jet break at $t \sim 10^{6} \mathrm{~s}$ to coincide with the break in the X-ray afterglow does not change the fits significantly.

The complicated behavior of the X-ray afterglow was explained by Racusin et al. (2008b) using a two-component jet model - a central narrow jet surrounded by a coaxial wider jet. In this model, the X-ray afterglow from the central narrow jet dominates at early times, until about $2 \times 10^{4} \mathrm{~s}$, when it fades below the brightness of the surrounding wide jet. In this model, the breaks in the X-ray afterglow light curves at times $t_{\mathrm{b}, \mathrm{X} 1} \sim 2 \times 10^{3} \mathrm{~s}$ and $t_{\mathrm{b}, \mathrm{X} 3} \sim 10^{6} \mathrm{~s}$ correspond to the jet breaks caused by the lateral spread of narrow and wide jets, respectively. We also attempted to reproduce the entire data set using this two-component-jet model, but found that with this model the optical radiation from the narrow jet would be brighter than the observed optical afterglow (see Fig. 2). There is no possible way to suppress this optical radiation from the narrow jet. The dominance of RS over
FS contribution at early times of the light curves are also shown for optical-NIR frequencies in Fig. 3 for both the models.

Alternatively, it is possible that the X-ray and optical afterglows of GRB 080319B are not related to each other and may have independent origins. The X-ray afterglow predicted by the $\mathrm{RS}-\mathrm{FS}$ model is also fainter than the observed one and requires an additional component.

Assuming that the shock expands into the circum-burst medium, which has a $W M$ density profile, our best-fit model spectral parameters at epoch $t=10^{4} \mathrm{~s}$ imply that the peak of the synchrotron spectrum is below the optical bands $\left(v_{\mathrm{m}}<1.3 \times\right.$ $\left.10^{14} \mathrm{~Hz}\right)$ with corresponding normalization flux $\left(F_{v_{\mathrm{m}}}<607 \mu \mathrm{Jy}\right)$ and the self-absorption frequency, $v_{\mathrm{a}}>10^{8} \mathrm{~Hz}$. The index of electron energy distribution $p \sim 2.07$ is lower than the canonical value $p \sim 2.3$, which has often been observed (Panaitescu \& Kumar 2001, 2002; Starling et al. 2008). The location of the cooling break frequency can be constrained to be below the $\mathrm{X}$-ray band $v_{\mathrm{c}} \leq 10^{18} \mathrm{~Hz}$, which would be consistent with the observed X-ray afterglow at late times $\left(t>10^{5} \mathrm{~s}\right)$. However, if the origin of the X-ray afterglow is independent of the optical afterglow, then $v_{\mathrm{c}} \ll 10^{18} \mathrm{~Hz}$. 



Fig. 1. Light curves of the GRB 080319B afterglow: the optical-NIR and $\mathrm{X}$-ray afterglow is shown in the top panel and the radio data in the bottom panel. The present optical observations are plotted along with other published optical and X-ray data. The FS-RS model is used to fit the afterglow light curves. The solid lines in the plots represent the resultant best fit WM model whereas dotted line show the best fit $I S M$ model.

The estimated isotropic equivalent afterglow kinetic energy $\left(E_{K}^{\text {iso }}\right)$ released in this explosion turns out to be $<5.5 \times 10^{53} \mathrm{erg}$. For the assumed $W M$ circumburst medium, we find the parameter value $A_{*}>0.01$. The fraction of the total energy given to accelerating electrons $\left(\epsilon_{\mathrm{e}}\right)$ and to the magnetic fields $\left(\epsilon_{B}\right)$ turns out to be about $>0.41$ and $<3 \times 10^{-3}$, respectively. The absence of any jet break until about $10^{6} \mathrm{~s}$ imply a jet opening angle $>1.4$ degrees, and hence the true amount of released energy must be $>8 \times 10^{49} \mathrm{erg}$. The radius of the fireball at $10^{4} \mathrm{~s}$ was $<5 \times 10^{18} \mathrm{~cm}$, which we extrapolated back in time to estimate the physical size of the emitting region during the optical flash $(t \sim 50 \mathrm{~s})$ and found to be $<3.6 \times 10^{17} \mathrm{~cm}$. Similarly, the Lorentz factor of the blast-wave was $<64$ at $10^{4} \mathrm{~s}$ after the burst, which corresponds to the Lorentz factor of about $<240$ at the time of the optical flash. These findings, which are only based on the evolution in the afterglow, are comparable with those of Racusin et al. (2008b) and Kumar \& Panaitescu (2008), who used arguments based on prompt emission to reach their conclusions. It is reassuring that the different approaches measured similar fireball sizes and blast-wave Lorentz factors.

The afterglow can also be explained by assuming ISM density profile of the circumburst medium. In this case, the best-fit model spectral parameters at epoch $t=10^{4} \mathrm{~s}$ were found to be $v_{\mathrm{m}}<2.1 \times 10^{14} \mathrm{~Hz}$ with corresponding flux $F_{v_{\mathrm{m}}}<566 \mu \mathrm{Jy}$,


Fig. 2. The afterglow of GRB 080319B - a comparison with the doublejet model: the model light curves shown in top and bottom panels are plotted for the WM and ISM cases, respectively. The model here assumes the double-jet scenario. The contribution to the afterglow only due to the narrow jet is shown in the figure. The narrow jet is assumed to be responsible for the early X-ray afterglow.

and the self-absorption frequency, $v_{\mathrm{a}}>10^{8} \mathrm{~Hz}$. To explain the relatively steep decay of the afterglow, the required value of $p=2.71$ is on a high side, and $v_{\mathrm{c}} \leq 10^{18} \mathrm{~Hz}$, as in the case of a $W M$ density profile.

The physical parameters estimated using these best-fit spectral parameters are $E_{K}^{\text {iso }}<2.4 \times 10^{52} \mathrm{erg}, \epsilon_{\mathrm{e}}>0.14, \epsilon_{B}<0.02$, and $n>7.5 \times 10^{-4}$ atoms/cc. The absence of any jet break until about $10^{6} \mathrm{~s}$ means that the jet opening angle of $\theta_{\mathrm{j}}>4.6 \mathrm{de}-$ grees, and hence the beaming corrected afterglow kinetic energy is $E_{\mathrm{K}}^{\mathrm{corr}}>3.8 \times 10^{49} \mathrm{erg}$. The radius of the fireball at $10^{4} \mathrm{~s}$ turns out to be about $<2.1 \times 10^{18} \mathrm{~cm}$ and at the time of the optical flash, $t \sim 50 \mathrm{~s}$, is about $<5.5 \times 10^{17} \mathrm{~cm}$. The corresponding Lorentz factors are $<52.4$ and $<382$, at $10^{4}$ and $50 s$ after the burst, receptively. As before, these values are also comparable to those of Racusin et al. (2008) and Kumar \& Panaitescu (2008).

\section{Conclusions}

We have presented observations of the GRB 080319B afterglow at radio, mm, and optical frequencies. The simultaneous multi-band afterglow modeling is useful in constraining the nature of the burst emission mechanism and the ambient medium. We found that the GRB 080319B afterglow is consistent with the fireball expanding either into the ISM or into the WM 

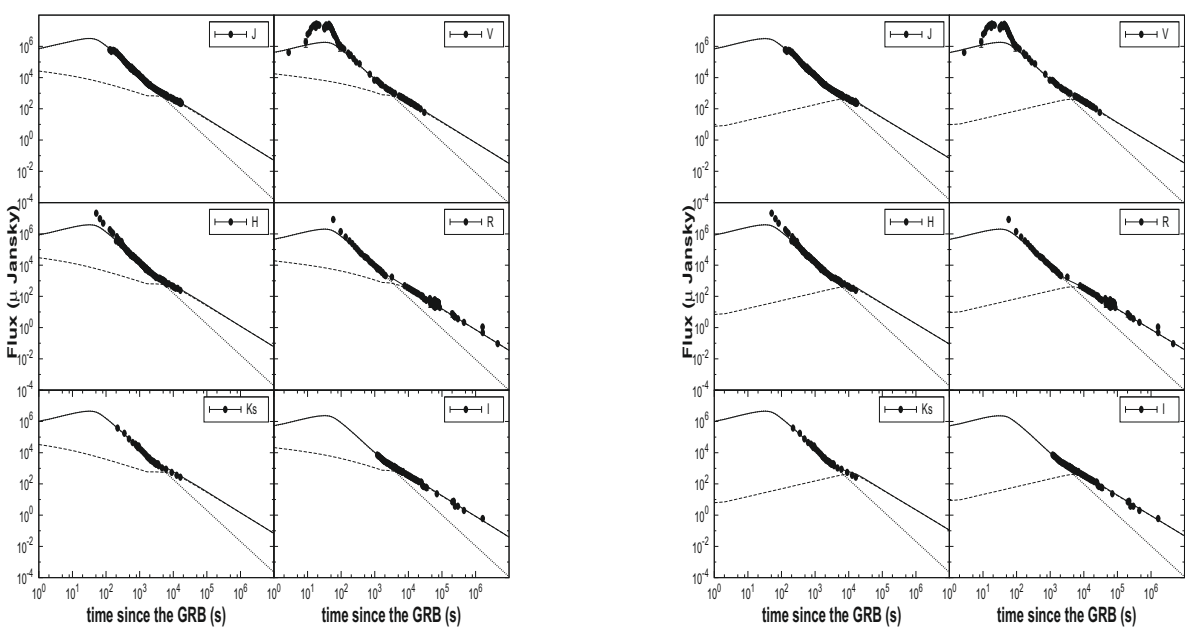

Fig. 3. The contributions by the RS and FS to the GRB 080319B afterglow at optical-NIR bands are represented by using the dashed and dotted lines, respectively. The addition of the two components is shown using the solid line. The left and right panels are WM and ISM models, respectively.

circumburst medium. We also excluded the double-jet model as a correct explanation of the observed multi-band behavior of the afterglow. Based on the assumptions of our model, we were able to explain the multi-band optical and radio afterglow reasonably well, but were unable to explain the X-ray afterglow. It is possible alternatively, that the optical and X-ray afterglows have different origins. The Lorentz factor of the shock was estimated from the afterglow evolution. The Lorentz factor at the time of naked-eye brightness, extrapolated from the late estimation, was found to be $\sim 300$. The corresponding radius of the shock front is about $10^{17} \mathrm{~cm}$. We also showed that the early peak brightness of the afterglow could not be caused the reverse shock. An additional emission mechanism, such as internal shocks, is required. Our results also indicate that existing blast-wave afterglow models should to modified in the light of the complicated behavior inferred for observed afterglows. In the future, observations of prompt optical spectra will be very useful in understanding the very early part of the afterglows of GRBs.

Acknowledgements. This research has made use of data obtained through the High Energy Astrophysics Science Archive Research Center On Line Service, provided by the NASA/Goddard Space Flight Center. Partly supported by the Spanish Ministry program AYA 2007-63677. SBP acknowledge the IndoRussian (DST-RFBR) project No. RUSP-836 (RFBR-08-02:91314) for this research work and Prof. V.V. Sokolov for the useful discussions. The coauthor V.C., a Research Fellow, acknowledges the Belgian National Fund for Scientific Research (FNRS) for the financial support. Observation in Ussuriisk Astrophysical Observatory was made with RS-22 telescope provided by ISON.

\section{References}

Akerlof, C., Balsano, R., Barthelmy, S., et al. 1999, Nature, 398, 400 Band, D., Matteson, J., Ford, L., et al. 1993, ApJ, 413, 281

Bloom, J., Perley, D. A., Li, W., et al. 2009, ApJ, 691, 723

Castro-Tirado, Alberto, J., Rosa Zapatero-Osorio, M., Caon, N., 1999, Science, 283, 2069

Chevalier, R. A., \& Li, Z. 2000a, ApJ, 520, L29

Chevalier, R. A., \& Li, Z. 2000b, ApJ, 536, 195

Cwiok, M., Dominik, W., MaÅek, K., et al. 2007, Ap\&SS, 309, 531

Cwiok, M., Dominik, W., Kasprowicz, G., et al. 2008, GCNC, 7439

Gehrels, N., Chincarini, G., \& Giommi, P. 2004, ApJ, 611, L1005

Gendre, B., Galli, A., Corsi, A., et al. 2007, A\&A, 462, 565

Golenetskii, S., Aptekar, R., Mazets, E., et al. 2008, GCNC, 7482

Gomboc, A., Guidorzi, C., Mundell, C. G. 2006, NCimB, 121, 1303
Granot, J., \& Sari, R. 2002, ApJ, 568, 820

Guilloteau, S., Delannoy, J., Downes, D., et al. 1992, A\&A, 262, 624

Henden, A. 2008, GCNC, 7528

Holland, S. T., \& Racusin, J. L. 2008, GCNC, 7496

Jelinek, M., Prouza, M., Kubanek, P. et al. 2006, A\&A, 454, L119

Kamble, A. P., Resmi, L., \& Misra, P. 2007, ApJ, 664, L5

Katz, J. I. 1994, ApJ, 422, 248

Klotz, A., Gendre, B., Stratta, G., et al. 2006, A\&A, 451, L39

Kobayashi, S. 2000, ApJ, 545, 507

Kumar, P., \& Panaitescu, A. 2008, MNRAS, 391, L19

Li, Z., \& Chevalier, R. A. 2001, ApJ, 551, 940

MacFadyan, A. I., \& Woosley, S. E. 1999, ApJ, 524, 262

Meśzáros, P. 2006, Rep. Prog. Phys., 69, 2259

Meśzaŕos, P., \& Rees, M. J. 1994, MNRAS, 269, L41

Meśzaŕos, P., \& Rees, M. J. 1997, ApJ, 476, 232

Meśzaŕos, P., \& Rees, M. J. 1999, MNRAS, 306, L39

Molinari, E., Bondar, S., Karpov, S., et al. 2006, Nuovo Cimento, 121, 1525

Nousek, J., Kouveliotou, C., Grupe, D., et al. 2006, ApJ, 642, 389

Panaitescu, A., \& Kumar P. 2001, ApJ, 560, L49

Panaitescu, A., \& Kumar, P. 2002, ApJ, 571, 779

Panaitescu, A., Meśzaŕos, P., \& Rees, M. J. 1998, ApJ, 503, 314

Pandey, S. B., Sahu, D. K., Resmi, L., et al. 2003, BASI, 31, 19

Price, P. A., Berger, E., Reichart, D. E., et al. 2002, ApJ, 572, L51

Piran, T. 1999, Phys. Rep., 314, 575

Racusin, J. L., Gehrels, N., Holland, S. T., et al. 2008a, GCNC, 7427

Racusin, J. L., Karpov, S. V., Sokolowski, M., et al. 2008b, Nature, 455, 183

Ramirez-Ruiz, E., \& Fenimore, E. E., 2000, ApJ, 539, 712

Rees, M. J., \& Meśzaŕos, P. 1992, MNRAS, 258, L41

Resmi, L., Ishwara, C. H., Castro-Tirado, A. J., et al. 2005, A\&A, 440, 477

Sagar, R. 2005, BASI, 33, 352

Sari, R., \& Piran, T. 1997a, ApJ, 485, 270

Sari, R., \& Piran, T. 1997b, MNRAS, 287, 110

Sari, R., Piran, T., \& Narayan, R., 1998, ApJ, 497, L17

Sari, R., Piran, T., \& Halpern, J. P. 1999, ApJ, 519 L17

Stamatikos, M., Ukwatta, T. N., Sakamoto, T., et al. 2009

[arXiv:0809.2132v1]

Stanek, K. J., Dai, X., Prieto, J. L., et al. 2007, ApJ, L21

Starling, R. L. C., Van Der Horst, A. J., Rol, E., et al. 2008, ApJ, 672, 433

Tanvir, N. R., Evert, R., Andrew, L., et al. 2009, ApJ, submitted [arXiv: 0812.1217]

Vestrand, W. T., Wozniak, P. R., Wren, J. A., et al. 2005, Nature, 435, 178

Vestrand, W. T., Wren, J. A., Wozniak, P. R., et al. 2006, Nature, 442, 172

Vreeswijk, P. M., Smette, A., Malesani, D., et al. 2008, GCNC 7451

Wang, J. H., Schwamb, M. E., Huang, K. Y., et al. 2008, ApJ, 679, L5

Wijers, R. A. M. J., \& Galama, T. J., 1999, ApJ, 523, 177

Wozniak, P. R., Vestrand, W. T., Panaitescu, A., et al. 2009, ApJ, 691, 495

Yu, Y. W., Wang, X. Y., \& Dai, Z. G. 2008 [arXiv:0806. 2010]

Zhang, B. 2007, Chin. J. Astron. Astrophys., 7, 1 